\title{
A model for soil moisture dynamics estimation based on artificial neural network
}

\author{
Wei Wang ${ }^{1,2}$, Shuya Wang ${ }^{3, *}$, Jianxia Chang ${ }^{1}$, and Dan Bai ${ }^{1}$ \\ ${ }^{1}$ State Key Laboratory Base of Eco-hydraulic Engineering in Arid Area, School of Water Resources \\ and Hydroelectric Engineering, Xi' an University of Technology, Xi' an 710048, China; \\ ${ }^{2}$ Xiaolangdi Project Construction \& Management Center, Ministry of Water Resources, Zhengzhou \\ 450000, China; \\ ${ }^{3}$ Henan Institute of Science and Technology, Xinxiang 453600, China
}

\begin{abstract}
Research on soil moisture estimation models can effectively improve the growth environment of crops. In this paper, the author studied the artificial neural network and variation pattern of soil moisture. Then, application of the model for water diversion estimation was explored based on artificial neural network. On this basis, an optimization algorithm was presented to simulate water diversion. Furthermore, a model for remote sensing of soil moisture dynamics was applied to artificial neural network. It has been proven that the research can optimize the application of the proposed model, laying a solid foundation for future study.
\end{abstract}

\section{Introduction}

Nowadays, there is a growing sense that water resources play a critical role in agriculture. As a measurement of water quantity in soil, soil moisture has a direct bearing on growth quality and production of crops. (Li 2006)The existing models for estimating soil moisture dynamics have been successful in indicating moisture gain and loss in the growth of crops. However, application of the models still remains to be improved, with a view to enhancing the growth quality of crops. Therefore, while estimating water demand for the growth of crops, the proposed model can save water and improve cultivation.

\section{Artificial neural network and variation pattern of soil moisture}

The widely-used artificial neural network forms in three layers, including data input layer, result output layer and hidden layer.(Qiu and Song and Wang et al, 2015) There exist different data exchange modes between two layers. In the computing of artificial neural network, a vector quantity and a weight vector need to be input to a neuron. At the same time, a nonlinear function is used for the measurement of neurons. In a single neuron, a hyperplane equation is utilized to separate the space into two parts and measure the given vector. The equation is defined as $\mathrm{Wp}+\mathrm{b}=0$, where $\mathrm{W}$ is a weight vector, $\mathrm{b}$ is the deviation

\footnotetext{
* Corresponding author: 232771432@qq.com
} 
value and $\mathrm{p}$ is the vector quantity in the hyperplane. Then, the vector value can be calculated using the equation.

As a key to establishing the model, the variation pattern of soil moisture is mainly concerned with time and space.

First, the temporal variation pattern means soil moisture changes over time. A survey found that soil moisture remains relatively stable and shows little changes all around the year, with August seeing the maximum. (Tang and Shen and Du et al, 2014)Besides, soil moisture is associated with underground depth of the soil layer. In general, the lower layer has greater soil moisture than the upper one.

Second, the spatial variation pattern means soil moisture changes with geographical locations. For example, overall soil moisture varies slightly in Kaifeng City of Henan Province. However, the parameter is much smaller in Minquan County of Henan Province, indicating a desperate lack of water quantity in soil. In contrast, the parameter in Yucheng County of Henan Province is apparently higher than other regions, suggesting that there exist greater differences in factors influencing soil moisture. (Zhang 2014) It is obvious that the model should be established based on the variation patterns of soil moisture. In this way, the established model can be stable and feasible, hence improving the model application. (Cheng 2017)

\section{Application of the proposed model based on artificial neural network}

\subsection{Design methods for the proposed model}

The design considered overall functions and system of the model. For one thing, overall functions mainly include system function module, data input module, data management and edition module, information release and management systems. (Deng 2016) In reality, systems interfaces should be entered from the user interface before entering function interfaces. Besides, each estimating item matches a corresponding data module. For example, graphic, statistical and descriptive data correspond to the data input module. Spatial database, attribute database and data dictionary belong to the data management and edition module. (Yuan and Duan and Chang et al,2016) The system function module relates to soil moisture dynamics and estimation modules based on RS, estimation module for cropland water demand and estimation module for general water demand in irrigated area. Moreover, the information release module involves table data and thematic map outputs, while the system management module is concerned with system maintenance and assistance. It can be seen that each module has different functions. For this reason, a systematic design is necessary to develop the model for water diversion estimation.(Feng 2015)

For another, in terms of system design, design objectives, based on spatial attribute and information, should be set in combination with such functions as information storage, processing and inquiry. As a result, a visualized and continuous model was built to provide the planter with the information on water consumption. ( $\mathrm{Hu}$ and $\mathrm{Li}, 2014) \mathrm{Then}$, the model for water diversion estimation was established according to the design principles, including sharing principle, the principle of stability, security principle and extensibility principle. In this paper, monthly water diversion estimation can be defined as the following regression equations.(Yang 2014, Ang and Shen and Du et al,2013) 
January

$$
\mathrm{W}_{1}=0.2457-0.0151 \mathrm{P}_{\mathrm{f} 4}+0.0235 \mathrm{R}_{\mathrm{fu}}(\mathrm{R}=0.835)
$$

February

$$
\mathrm{W}_{2}=-1.0448+0.6004 \mathrm{Rf}-0.0705 \mathrm{P}_{\mathrm{f} 3}(\mathrm{R}=0.842)
$$

March

$$
\mathrm{W}_{3}=17.9212+1.0804 \mathrm{~W}_{\mathrm{u}}+0.4712 \mathrm{R}_{\mathrm{t}}+0.3342 \mathrm{RH}(\mathrm{R}=0.953)
$$

April

$$
\mathrm{W}_{4}=11.8294+0.6426 \mathrm{R}_{\mathrm{u}}-0.1462 \mathrm{ET}_{0 \mathrm{u}}(\mathrm{R}=0.837)
$$

May

$$
\mathrm{W}_{5}=1.1181+0.5122 \mathrm{~W}_{\mathrm{u}}(\mathrm{R}=0.684)
$$

June

$$
\mathrm{W}_{6}=16.1685-0.0795 \mathrm{R}_{\mathrm{fu}}+0.9508 \mathrm{R}_{\mathrm{f}}-0.8841 \mathrm{R}_{\mathrm{t}}(\mathrm{R}=0.839)
$$

July

$$
\mathrm{W}_{7}=11.0354-0.1254 \mathrm{~W}_{\mathrm{f} 4-0.0248 \mathrm{P}_{\mathrm{f} 4}(\mathrm{R}=0.700)}
$$

August

$$
\mathrm{W}_{8}=11.4216-0.0369 \mathrm{P}_{\mathrm{f} 2}(\mathrm{R}=0.907)
$$

September

$$
\mathrm{W}_{9}=-68.7867+0.6232 \mathrm{ET}_{0}-0.1673 \mathrm{~N}+1.7311 \mathrm{~T}_{\mathrm{u}}(\mathrm{R}=0.933)
$$

October

$$
\mathrm{W}_{10}=9.9036-0.0242 \mathrm{P}_{\mathrm{f} 2}-0.0325 \mathrm{P}_{\mathrm{u}}(\mathrm{R}=0.784)
$$

November

$$
\mathrm{W}_{11}=7.6075-0.1177 \mathrm{RH}+0.0510 \mathrm{~W}_{\mathrm{f} 4}(\mathrm{R}=0.720)
$$

December

$$
\mathrm{W}_{12}=-97.5909+0.1121 \mathrm{P}_{\mathrm{a}}+1.1025 \mathrm{~W}_{\mathrm{u}}(\mathrm{R}=0.794)
$$

\subsection{Application of the proposed model}

Application of the proposed model mainly comprises irrigation data processing, system file management and functions for map processing and inquiry. First, in irrigation data processing, control points need to be selected following the principles of uniform distribution, recognition and accuracy of control points as well as data vectorization. (Zheng 2013) Then, the system file management is mainly concerned with maps or layers 
used in the model, including text addition, text saving and image saving. In the process of management, a comprehensive system control is implemented using the control layer. At last, map processing, including zooming in/out, roam and overall view, can be realized by clicking the system menu. (Wang 2011)

\subsection{Optimization algorithm for water demand}

In the proposed model, there are three major optimization algorithms, including singlefactor linear regression, multi-factor linear regression and projection pursuit regression. In reality, single-factor linear regression used to calculate the efficiency coefficient is characterized by effective simulation effect and high accuracy. The multi-factor linear regression is utilized to analyze the influence of multiple factors on final results. As a new form of data analysis, projection pursuit regression can deal with the distribution of nonlinear and non-normal data, making a difference in practical application.

\section{Remote sensing of soil moisture dynamics on artificial neural network}

\subsection{Monitoring methods for soil moisture}

In the model for remote sensing and estimation of soil moisture dynamics, remote sensing is mainly concerned with the recognition of crop area and distribution, the recognition of water quantity in soil as well as the establishment, verification and application of the monitor model. Remote-sensing image pretreatment is a major measure for remote sensing. Satellite data with high resolution and short time period is collected to acquire remotesensing images, which can mirror the spectral signatures of different crops based of their properties, thus effectively avoiding isospectrality. Pretreatment software can be employed to get data. For instance, in a guided process, ENVI software can be used for atmospheric correction in a short time, so as to enhance the quality of soil moisture monitoring. While reducing the effects of the atmosphere on data, atmospheric correction can process hyperspectral, satellite and aeronautical data as well as correct the vertical imaging. The index for soil moisture monitoring can be formulated as:

$$
\mathrm{CI}=\mathrm{SM} / \mathrm{SSM}
$$

where $\mathrm{C}$ is the index for soil moisture monitoring, SM is the actual water quantity in soil and SSM is the appropriate water quantity for crops.

\subsection{Establishment of the remote sensing model for soil moisture dynamics}

Establishing the remote sensing model needs to analyze the methods for monitoring water quantity in agricultural and hydraulic sectors. Additionally, soil layer in crop root was fully analyzed based on remote sensing image of the satellite. For example, to define the index for soil moisture monitoring, there are two real-time indicators, namely the actual water quantity in soil and the appropriate water quantity for crops. The latter varies with types of crops, growth cycle and water supply. The upper and lower limits of appropriate water quantity in soil can be used as the monitoring indexes. Water moisture in soil via remote sensing can be described as: 


$$
\mathrm{RSM}=\mathrm{RSM}_{\mathrm{W}}-\mathrm{TVDI}^{*}\left(\mathrm{RSM}_{\mathrm{W}}-\mathrm{RSM}_{\mathrm{D}}\right)
$$

where RSW is the relative water quantity in soil, RSMW is the maximum relative water quantity in soil and is RSMD the minimum relative water quantity in soil.

\subsection{Software development in the remote sensing model for soil moisture dynamics}

The software should integrate a wide range of functions, including processing of monitoring images, index calculation, calculation of water quantity in soil, monitoring of water moisture and browse of thematic graphs. In the design of software user and interfaces, user needs to input or obtain information from the interfaces. On this basis, it is essential to secure information and avoid the user getting access to confidential information in the proposed model. In addition, data category lays the basis for designing date layer. The backend data layer refers to database system and centralized information storage system, making it possible to store many kinds of information. Application of software in the proposed model can be ensured only when the data system is consistent with the server. It can be seen that application of the proposed model needs to start with diverse models for soil moisture dynamics estimation, which can optimize the application of the model for soil moisture dynamics estimation and promote the development of future study.

\section{Conclusions}

Models for oil moisture dynamic estimation have attracted more and more attention. It follows, therefore, that researchers have attached greater importance on how to enhance the quality of model application and improve the planting of crops. This paper found that artificial neural network can optimize the application of the proposed model, laying a solid foundation for future study.

\section{References}

1. SH. Li,Chinese Academy of Agricultural Sciences (2016)

2. MJ. Qiu, YB. Song, JL.Wang, Chinese Journal of Agrometeorology; 2,187-94 (2015)

3. CY.Tang, SH. Shen, YL. Du, Acta Agriculturae Universitatis Jiangxiensis, 35,1082-1089(2014)

4. XF. Zhang, Anhui Agricultural University(2014)

5. AZ. Cheng, Agricultural engineering technology, 29 (2017)

6. YZ. Deng, Qinghai Agro-Technology Extension, 4,52-53(2016)

7. JF. Yuan, L. Duan, NN. Chang, farmer rich, 16 (2016)

8. YH. Feng, Water ecological security -- the 2015 BBS excellent essay collection of water resources peak, 9 (2015)

9. XY. Hu, ZZ. Li, soil and crop, 3,99-104(2014)

10. HM. Yang,Zhengzhou University(2014)

11. CY. Ang, SH. Shen, XL. Du, XZ. Qiu, XY. Sun, Journal of the Jiangxi Agricultural University, 35, 1082-1089(2013)

12. QH. Zheng, Shihezi University( 2013)

13. XL. Wang, Zhengzhou University(2011) 